\title{
Review Article \\ Underappreciated Consequences of Phenotypic Plasticity for Ecological Speciation
}

\author{
Benjamin M. Fitzpatrick \\ Ecology and Evolutionary Biology, University of Tennessee, Knoxville, TN 37996, USA \\ Correspondence should be addressed to Benjamin M. Fitzpatrick, benfitz@utk.edu \\ Received 25 July 2011; Revised 22 October 2011; Accepted 12 December 2011 \\ Academic Editor: Andrew Hendry
}

Copyright ( $) 2012$ Benjamin M. Fitzpatrick. This is an open access article distributed under the Creative Commons Attribution License, which permits unrestricted use, distribution, and reproduction in any medium, provided the original work is properly cited.

\begin{abstract}
Phenotypic plasticity was once seen primarily as a constraint on adaptive evolution or merely a nuisance by geneticists. However, some biologists promote plasticity as a source of novelty and a factor in evolution on par with mutation, drift, gene flow, and selection. These claims are controversial and largely untested, but progress has been made on more modest questions about effects of plasticity on local adaptation (the first component of ecological speciation). Adaptive phenotypic plasticity can be a buffer against divergent selection. It can also facilitate colonization of new niches and rapid divergent evolution. The influence of nonadaptive plasticity has been underappreciated. Non-adaptive plasticity, too can interact with selection to promote or inhibit genetic differentiation. Finally, phenotypic plasticity of reproductive characters might directly influence evolution of reproductive isolation (the second component of ecological speciation). Plasticity can cause assortative mating, but its influence on gene flow ultimately depends on maintenance of environmental similarity between parents and offspring. Examples of plasticity influencing mating and habitat choice suggest that this, too, might be an underappreciated factor in speciation. Plasticity is an important consideration for studies of speciation in nature, and this topic promises fertile ground for integrating developmental biology with ecology and evolution.
\end{abstract}

\section{Introduction}

Phenotypic plasticity has often been seen primarily as an alternative to genetic divergence and a feature making populations less responsive to natural selection [1-3]. For example, those studying adaptation and speciation have often used phrases like "merely plastic" to contrast environmentally induced variation against geographic or species differences with strong genetic bases [4-8]. However, others suggested that phenotypically plastic traits can promote adaptive evolution and the origin of species [9-16]. The general issue of plasticity and adaptation has been reviewed extensively in the last decade (e.g., [15, 17-26]). Adaptive plasticity's impact on speciation was recently reviewed by Pfennig et al. [27], and I do not attempt to duplicate their efforts. Instead I make a few points that have not been emphasized in the recent literature. In particular, nonadaptive plasticity and environmentally induced barriers to gene flow deserve greater attention.

After making explicit my working definitions of key terms, I argue that the "developmental plasticity hypothesis of speciation" [13-15] is a special case of ecological speciation, and I review the subject by breaking down the effects of plasticity on the two components of ecological speciation: adaptive divergence and the evolution of reproductive isolation [28]. I close with a few suggestions for future work.

By any definition, speciation requires genetic divergence. Therefore, integration of ecological developmental biology with the well-developed body of fact and theory on the genetics of speciation [29-31] will be more productive than attempting to replace this population genetic foundation. Recent reviews and models support this perspective [27, 3237].

\section{Definitions}

Understanding the relationship between environmental induction and speciation requires a set of consistent definitions. Terms like "environment," "speciation," "plasticity," and "natural selection" are sometimes assumed by 
different workers to have different definitions, and this can affect communication [38]. The definitions that follow are intended to clarify what I mean by certain words and phrases within this paper; they are not intended to challenge or replace alternative definitions. I believe I have followed recent convention in all cases $[39,40]$.

Adaptation. Genetic change in response to natural selection and resulting in organisms with improved performance with respect to some function or feature of the environment.

Countergradient Variation. Pattern of geographic variation in which genetic differences between populations affect their phenotypes in the opposite way from environmental differences between populations. For example, if the mean phenotype of population $i$ is given by the sum of genetic and environmental effects $P_{i}=G_{i}+E_{i}$, and the effect of environment 2 tends to increase the phenotypic value relative to environment $1\left(E_{2}>E_{1}\right)$, countergradient variation would exist if $G_{2}<G_{1}$. Without genetic differentiation the expected difference in phenotype would be $P_{2}-P_{1}=E_{2}-E_{1}$, but countergradient variation reduces that difference and might even make $P_{2}<P_{1}$. More formally, countergradient variation is negative covariance between genetic and environmental effects on phenotype [41].

Environment. Here, I consider environment to include anything external to a given individual organism. Environment includes other organisms (siblings, mates, competitors, predators, prey, etc.) in addition to the physical and chemical surroundings. Given this definition, different individuals in the same place might experience different environments. Neither ecological speciation nor environmentally induced variation require environmental differences to be associated with geography. To put it another way, the effects of environment on fitness and on development might differ among cooccurring individuals for a variety of reasons, often involving feedbacks between phenotype and environment [42]. For example, small tadpoles in a pond might experience food shortages or attacks from predators while large tadpoles in the same pond have access to more food and experience less predation (or a different set of predators).

Environmental Induction. Any effect of environment on trait expression is environmental induction. Environmental induction usually refers to an event (developmental outcome or process) caused by an environmental condition, whereas plasticity (see below) refers to the propensity of an organism or trait to respond to environmental change.

Genetic Assimilation. Evolutionary reduction in the degree of plasticity such that a character state or trait value that was once conditionally expressed depending on the environment becomes expressed constitutively (unconditionally, regardless of environment).

Natural Selection. Natural selection refers to any nonrandom difference between entities in survival or reproduction.
To put it another way, natural selection exists whenever phenotypic variation causes covariance between phenotype and fitness $[43,44]$. Fitness is metaphorical shorthand for the ability to survive and reproduce. It is important to emphasize that natural selection, under this definition, can exist without genetic variation and can recur over many generations without causing evolution $[15,17]$.

Plasticity. The ability of a single genotype to express different phenotypic values or states under different environmental conditions, that is, in response to environmental induction. Plasticity can include developmental plasticity, physiological acclimation, or behavioral flexibility. Plasticity might be adaptive or not. Adaptive plasticity is a tendency for a genotype to express a phenotype that enhances its ability to survive and reproduce in each environment. Nonadaptive plasticity includes any response to environmental induction that does not enhance fitness (including maladaptive responses). Noisy plasticity is effectively unpredictable phenotypic variation owing, for example, to developmental instability or random perturbations within environments [45]. Phenotypic plasticity and environmental induction are twin concepts; plasticity emphasizes an organismal property (the propensity to express different phenotypes in different environments), and environmental induction emphasizes the action of the environment. Phenotypic plasticity might exist even in a homogeneous population in a homogeneous environment. Environmental induction happens when environmental heterogeneity causes phenotypic heterogeneity.

Polyphenism. Expression of more than one discrete phenotypic state (alternative phenotypes) by a single genotype (a special case of phenotypic plasticity).

Reaction Norm [or Norm of Reaction]. The set of expected phenotypic states or values expressed by a genotype over a range of environments.

Speciation. Speciation is any process in which an ancestral species gives rise to two or more distinct descendant species. There is some disagreement about whether "speciation" should be synonymous with the evolution of reproductive isolation $[30,46,47]$ or broadened to include anagenesis or phyletic speciation [48-50]. In any case, speciation is usually a gradual, continuous process of genetic divergence resulting in a discontinuous pattern of variation (species taxa). Ecological speciation is the evolution of reproductive isolation as a consequence of divergent ecological adaptation $[28,51,52]$. Without reproductive isolation, this is local adaptation. This definition is based on the biological species concept [53], which emphasizes genetically based reproductive isolation as the primary explanation for the existence of distinct kinds of organisms (i.e., those recognized as species taxa). I am not making a recommendation about taxonomic practice. Rather, from the perspective of evolutionary biology, the evolution of reproductive isolation is what distinguishes speciation from more general phenomena of genetic divergence $[30,40]$. 


\section{The Developmental Plasticity Hypothesis of Speciation}

Matsuda [54] hypothesized that phenotypic plasticity was a crucial first step in the adaptive evolution of distinct, ecologically specialized lineages. As an example, Matsuda [54] suggested that major differences in life history, such as presence or absence of metamorphosis prior to reproduction in salamanders (Figure 1), likely began as nongenetic polyphenisms and evolved via genetic assimilation in habitat specialists. Widespread generalists such as Ambystoma tigrinum and $A$. velasci show conditional expression of metamorphosis from aquatic larva to terrestrial adult in small temporary ponds versus a fully aquatic life cycle with no metamorphosis in more permanent water bodies. This polyphenism likely represents the ancestral state of the tiger salamander clade [55]. In several isolated lakes in Mexico, permanently aquatic endemics such as A. mexicanum and A. dumerilii no longer express metamorphosis in nature owing to genetic changes in the thyroid hormone system [54-56].

Along the same lines, West-Eberhard [13-15] proposed a generalized "developmental plasticity hypothesis of speciation" in which the evolution of ecologically distinct forms in different environments depends on the initial appearance of those distinct forms as alternative phenotypes in a phenotypically plastic ancestor. She argued that when adaptive phenotypic plasticity results in strong associations between phenotypes and environments, rapid speciation could occur in three steps. First, alternative phenotypes become fixed in different populations owing to environmental differences, but with little or no genetic change. Then, genetic assimilation and/or other adaptive modifications of each phenotype occur owing to divergent selection. Finally, reproductive isolation evolves as a byproduct of adaptive divergence or via reinforcement if there is contact between the diverging populations.

Clearly, West-Eberhard's [13-15] hypothesis is a kind of ecological speciation in which developmental plasticity promotes genetic divergence in response to ecologically based selection. In the absence of plasticity, divergence might be prevented entirely if the single expressed phenotype cannot establish a viable population in the alternative environment $[22,27,36]$ or might be much slower if phenotypic divergence must await new mutations and their gradual fixation [13-15].

West-Eberhard's developmental plasticity hypothesis of speciation is focused on adaptive phenotypic plasticity and its influence on one component of ecological speciation: the evolutionary response to divergent selection. However, nonadaptive plasticity might be equally if not more influential in promoting an evolutionary response [18]. Further, the other component of ecological speciation, the evolution of reproductive isolation $[28,62,63]$, also can be directly influenced by phenotypic plasticity. In the next sections, I examine how plasticity can interact with these two components of ecological speciation.

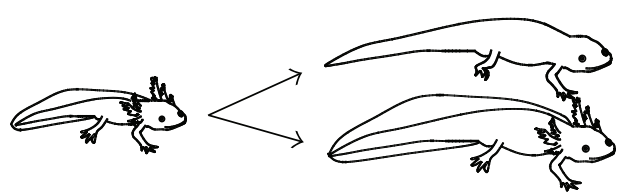

(a)

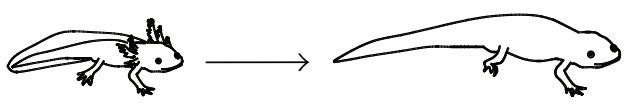

(b)

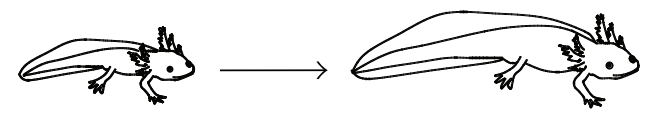

(c)

FIGURE 1: The tiger salamander radiation exemplifies the developmental plasticity hypothesis of ecological speciation [13-15, 54]. (a) Environmentally induced alternative phenotypes of the tiger salamander (Ambystoma tigrinum) include terrestrial metamorphs and aquatic paedomorphs. (b) The California tiger salamander (A. californiense) is an obligate metamorph derived from a developmentally plastic common ancestor with A. tigrinum [55]. (c) The Mexican axolotl (A. mexicanum) is one of several obligate paedomorphs, independently derived from plastic ancestors within the last few million years $[55,57,58]$.

\section{Phenotypic Plasticity and Ecological Divergence}

Phenotypic plasticity can slow or enhance genetic divergence. How plasticity affects divergence depends to some extent on whether plasticity is adaptive or not.

4.1. Adaptive Plasticity. Adaptive plasticity can dampen or eliminate divergent selection. If any individual can express a nearly optimal phenotype in whatever environment it finds itself, then there is little or no variation in the ability to survive and reproduce, hence little or no divergent selection [64]. This has long been an intuitive reason to regard plasticity as a constraint on genetic evolution and to discount the evolutionary potential of environmentally induced variation [1]. Models have supported the prediction that adaptive plasticity can effectively take the place of genetic divergence between environments [65-67]. And a large number of empirical studies are consistent with increased plasticity in species with high dispersal rates [68]. However, the extent to which plasticity prevents or slows genetic divergence depends on several factors explored by ThibertPlante and Hendry [36] in individual-based simulations.

First, is development sufficiently flexible that an individual can express traits near either environmental optimum? Given alternative environments or niches with divergent fitness functions, the only way environmental induction can completely eliminate divergent selection is to cause the mean expressed trait of a single gene pool to match the optimum in each environment [22] (Figure 2). If adaptive plasticity is less than perfect, divergent selection might still exist. Then the question is whether plasticity quantitatively dampens 


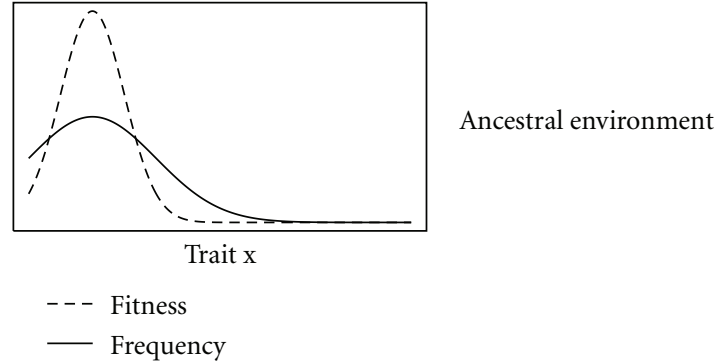

(a)

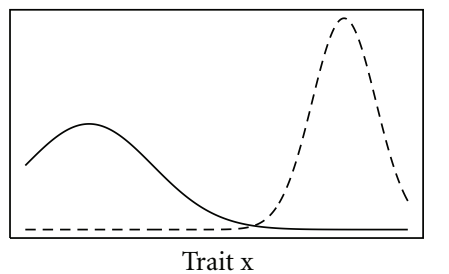

Zero plasticity:

High chance of extinction

in new environment

(b)

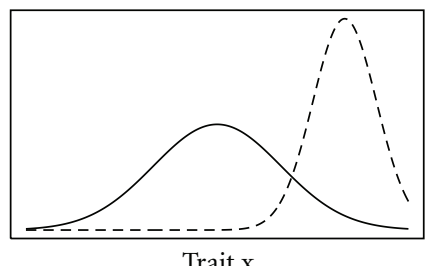

Moderate plasticity:

Facilitates colonization

and divergent selection

in new environment

(c)

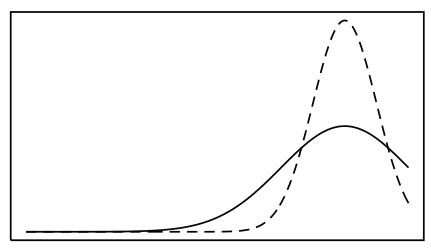

Strong plasticity: Prevents divergent selection

(d)

FIgURE 2: Effects of adaptive plasticity on colonization and adaptation to a new niche. A population well adapted to its niche is illustrated in (a) by coincidence of the mean trait value (black line) with the peak of the fitness function (dashed line). If the fitness function is dramatically different in a new environment, a population with trait values favored in the old environment (b) might have such low fitness as to have little chance of survival. If environmental induction produces a shift in trait values toward higher fitness phenotypes (c), the population might persist but still experience selection. If phenotypic plasticity results in a perfect match between mean trait value and fitness optimum (d), then there is no effect of selection on the population mean.

the fitness tradeoff enough to substantially slow or prevent divergent evolution [36].

Second, how much dispersal occurs between environments? In the absence of gene flow, almost any amount of divergent selection will eventually cause evolutionary divergence. When there is gene flow between populations, the effect of divergent selection depends on the relative magnitudes of selection and gene flow [1,69-73], in addition to demographic factors [74-76]. Therefore the impact of adaptive plasticity on the potential for genetic divergence depends on how it affects the tension between divergent selection and gene flow $[36,77,78]$. Moreover, plasticity itself is adaptive only to the extent that individuals have a reasonable chance of experiencing alternative environments. If the populations expressing alternative phenotypes are isolated in their respective environments, the ability to express the alternative phenotype is likely to be lost owing to selection for efficient development or simply because loss of function mutations are likely to accumulate neutrally in genes that are never expressed [79]. This process of a conditionally expressed trait becoming constitutively expressed is genetic assimilation $[11,16,80]$.

Third, are the systems sensing the environment and regulating trait expression sufficiently accurate that the best phenotype is reliably expressed in each environmental context? There are two components to this, first is simply the question of how well developmental or behavioral systems are able to sense and react to environmental stimuli [81]. Again, if adaptive plasticity is less than perfect, divergent selection can exist. Second is the question of whether the timing of key developmental and life history events is such that future environmental conditions can be correctly predicted [36]. For example, if individuals disperse and settle before completing development (e.g., seeds or planktonic larvae), they might be able to accurately tune their adult phenotypes to the environment in which they settle. However, if a substantial number of individuals disperse after completing development (e.g., animals with extended parental care [82]), then developmental plasticity would do little to help them accommodate new environmental challenges because their phenotypes are adjusted to their natal habitat rather than their new habitat. In this case, there might be strong selection against immigrants before any genetic differences arise between populations [36].

Finally, is there any cost to plasticity? Several modeling studies have confirmed the idea that plasticity is less likely to evolve if there are fitness costs to maintaining multiple developmental pathways or changing expression during development [36]. Empirical tests for costs of plasticity itself are rare [83], but it is conceivable that some pathways might have inherent tradeoffs between efficiency and plasticity $[84,85]$, and adaptive plasticity probably always comes with some potential for error; that is, the best developmental "decision" might not be made every time $[64,81]$. When plasticity is costly enough to outweigh its fitness benefits, possible alternative outcomes are the evolution of a single "compromise" or generalist phenotype, evolution of a simple genetic "switch" enabling coexistence of alternative specialist phenotypes $[14,15,86]$, or divergent evolution of specialist populations (local adaptation) [45, 67, 87]. In general we know very little about the prevalence or influence of costs of plasticity in nature.

The potential for adaptive plasticity to evolve as a response to ecological tradeoffs instead of genetic divergence is well supported. The dampening effect of plasticity is reduced but not eliminated by reduction in the extent and precision of plasticity, reduction in gene flow between environments, 


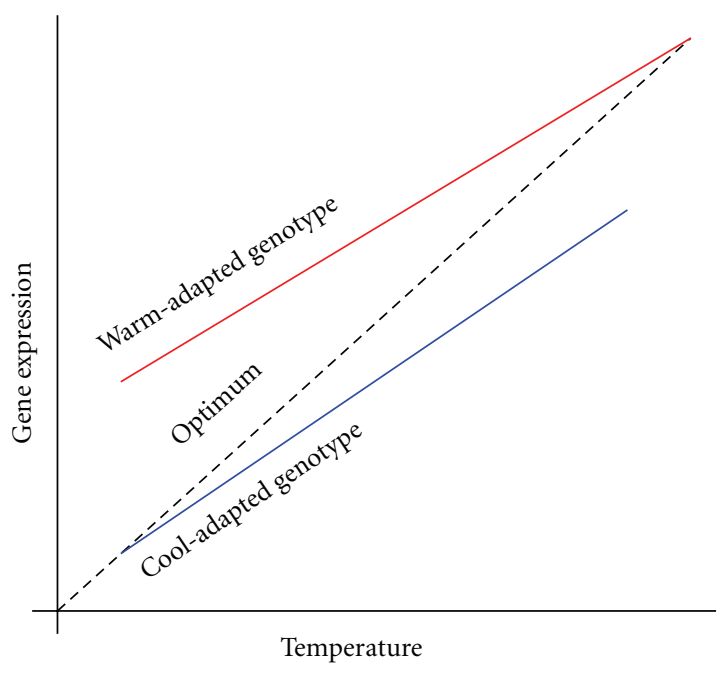

(a)

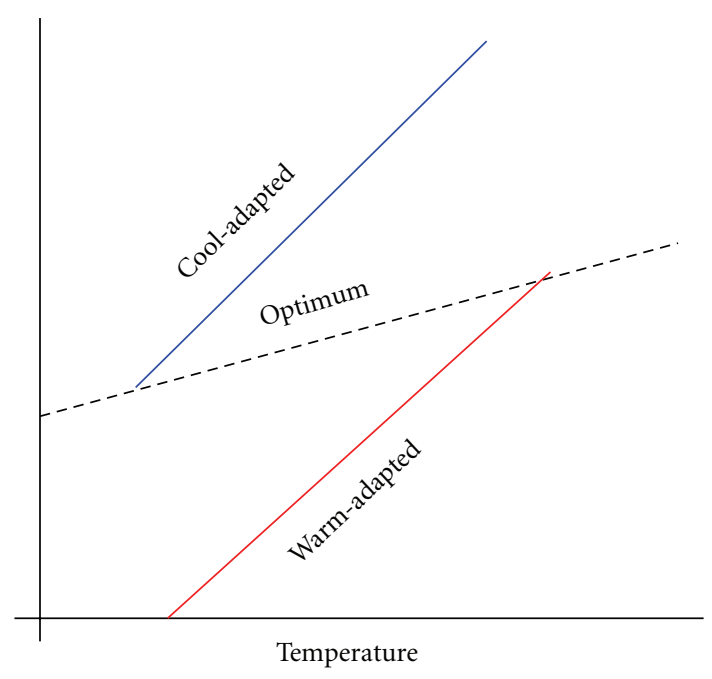

(b)

FIgURE 3: Adaptive and nonadaptive reaction norms. These hypothetical examples suppose that the optimum expression level for some gene increases with temperature and that increased temperature induces increased expression of the gene. (a) Plasticity is adaptive when it keeps expression closer to the optimum than it would be if expression, were constant across temperatures. (b) Plasticity is nonadaptive (maladaptive, in this case) when induced changes in a new environment take expression further from the optimum than it would be if it had remained constant. (b) is an example of countergradient variation, in which genetic differences cause cool-adapted genotypes to have higher gene expression than warm-adapted genotypes at the same temperature. The result is overexpression by cool-adapted genotypes transplanted to warm environments.

and increased costs of plasticity [36]. However, plasticity can actually promote genetic divergence under some conditions. In particular, when development is completed after dispersal (e.g., sessile organisms), adaptive plasticity might make successful colonization of new environments more likely (Figure 2). In West-Eberhard's [14, 15] conceptual model and Thibert-Plante and Hendry's [36] mathematical model, individuals are able to colonize a radically new environment by adjusting developmentally, behaviorally, and/or physiologically. This adaptive plasticity allows a population to persist in the new environment, continually exposed to divergent selection. If instead all individuals entering the new environment die or leave, there is no divergent selection. Thus, without adaptive plasticity, there might simply be suitable and unsuitable environments, with little opportunity for divergent evolution. Successful colonization of a new environment can initiate divergent selection, not only on the plastic trait, but possibly also on other traits, which might then cause ecological speciation. This to some extent reconciles the conflicting effects of plasticity. In other circumstances, when dispersal occurs after development (e.g., animals with extended parental care), individuals settling in new environments are especially likely to express suboptimal phenotypes, which might accentuate the effects of selection and spatial separation once a new habitat has been colonized [36]. This effect is similar to effects of nonadaptive plasticity discussed below.

4.2. Nonadaptive Plasticity. The potential for adaptive plasticity to promote colonization and adaptation to alternative environments has been promoted by advocates of developmental evolutionary biology $[14,15,17,88]$ and treated extensively in recent reviews and models [22, 27, $32,36]$. The effects of nonadaptive phenotypic plasticity have received less attention. However, any environmental effect on phenotypes can affect the strength and direction of selection in addition to the genetic variances and covariances of important traits $[18,22,42]$. Some kinds of nonadaptive environmental induction might affect the probability of ecological speciation. In particular, suboptimal development or noisy plasticity [45] in stressful environments could inhibit adaptation by decreasing the fitness of local relative to immigrant individuals. However, it would also increase the strength of selection and potentially result in cryptic adaptive divergence (countergradient variation) $[18,41]$.

Countergradient variation is negative covariance between genetic and environmental effects on phenotype [41]. Classic examples are poikilotherms such as fish and molluscs [89], flies [90], or frogs [91] that grow more slowly in cold climates, but cold-adapted populations have higher growth rates than warm-adapted populations when raised at the same temperature. The best explanation for this pattern is that genetic differences have evolved to compensate for divergent effects of environmental induction, resulting in populations that appear similar when measured each in their native habitat but show maladaptive plasticity when transplanted (Figure 3). Note that negative covariance between genetic and environmental effects is not necessarily maladaptive, but environmental effects will tend to be maladaptive if the optimum phenotype is roughly constant across the environment range. 
When countergradient variation exists, we expect immigrants to have a fitness disadvantage owing to under- or overexpression of an environmentally sensitive trait relative to a local optimum (Figure 3). This might well promote evolution of restricted or nonrandom dispersal; hence intrinsic barriers to gene exchange as a result of divergent ecological selection. However, countergradient variation will evolve only if selection is stronger than gene flow and if reaction norms are genetically constrained (otherwise, we might expect adaptive evolution to flatten the reaction norm). At least in the early stages of colonization of a challenging new habitat, nonadaptive plasticity (such as stunted growth or suboptimal metabolic rates) might make resident individuals less viable and fecund than healthy immigrants from a less stressful habitat [22]. This potential fitness asymmetry could offset effects of adaptive genetic changes on the relative fitness of immigrants and residents. That is, offspring of immigrants might have lower fitness than offspring of native genotypes with locally adaptive alleles. However, offspring of immigrants might nevertheless outnumber offspring of natives if immigrants come into the stressful habitat with substantial viability and fertility advantages from being raised in a higher quality habitat. Parental care and physiological maternal effects could further extend those environmentally induced advantages to the offspring. The net effect could be a tendency for locally adapted genotypes to be replaced ("swamped") by immigrants owing to a negative covariance between environmental and genetic effects. This effect of nonadaptive plasticity is synergistic with the potential for demographic swamping, a well-known constraint on local adaptation to novel habitats [74, 76, 87, 92].

For now, it appears that the impact of nonadaptive plasticity on the probability of ecological speciation cannot be predicted without additional detailed knowledge. Just as gene flow can constrain or facilitate local adaptation $[74,76]$, and adaptive plasticity can inhibit or promote adaptive genetic divergence $[22,27,36]$, nonadaptive plasticity might impede genetic divergence by accentuating fitness advantages of immigrants and/or promote divergence by increasing the intensity of selection.

\section{Phenotypic Plasticity and the Evolution of Reproductive Isolation}

Plasticity of a different sort might directly affect reproductive compatibility between populations developing in different environments. Environmental induction might generate differences in preference, reproductive phenology, or expression of secondary sexual characteristics. Coincidence of environmentally induced reproductive barriers and potentially divergent selection can be genetically equivalent to a geographic barrier between divergent environments $[59,93,94]$. A key element of the developmental plasticity model of ecological speciation is the establishment of a consistent relationship between the environment of parents and that of their offspring. Similarity of parent and offspring environments maintains shared environmental effects on phenotype, consistency of selection, and reduces gene flow.

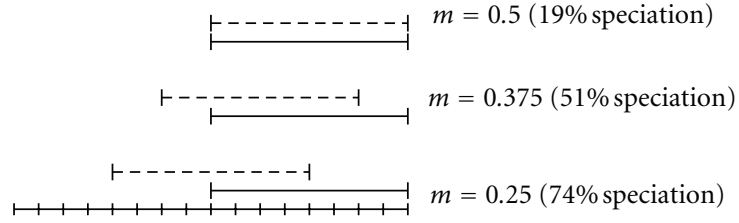

Flowering week

FIGURE 4: Effects of phenotypic plasticity of flowering time on ecological speciation. The lines illustrate flowering periods for plants on two soil types (dashed and solid) based on initial conditions in the simulations of Gavrilets and Vose [59] for ecological speciation based on the Howea palm tree study [60]. I illustrate the case where flowering time is affected by 8 loci, and the environmental effect of soil type is either 0 , 2, or 4 weeks difference. In the simulations [59], changing the number of loci has confounding effects on the initial environmental variance component and the fitness effects of mutations, so a single genetic scenario is illustrated here for simplicity. I calculated the initial rate of gene flow between soil types as $m=0.5$ (\% time overlap), based on the assumption that any tree is equally likely to receive pollen from any other currently flowering tree. \%speciation is based on the number of simulations ending in speciation, given in Table 2 of Gavrilets and Vose [59].

Environmental similarity can be a byproduct of geographic isolation or might be promoted by environmentally induced variation in habitat choice, phenology, or other aspects of mating behavior. Whether or not ecological speciation ensues depends on whether genetic reproductive barriers evolve and whether that evolution can be attributed to divergent selection.

A seemingly commonplace example of environmentally induced barriers to gene flow is flowering time in plants. Flowering time is often accelerated or delayed when a given genotype is grown on different substrates [34]. For example, grasses and monkey flowers colonizing contaminated soils around mines show environmentally induced shifts in flowering $[95,96]$, and palm trees on Lord Howe Island show soil-dependent flowering times [60]. These examples are also widely recognized cases of ecological speciation. Gavrilets and Vose [59] used simulations to explore the impact of environmentally induced shifts in flowering time on the probability of adaptive divergence and ecological speciation and confirmed that this instantaneous barrier to gene flow between habitats can markedly increase the probability and rate of divergence (Figure 4).

A similar effect arises owing to behavioural imprinting [97, 98]. Many animals, such as birds and anadromous fishes, are known for imprinting on their natal habitat [99-101]. When this is a direct matter of memorizing where home is (as might be the case in Ficedula flycatchers [101]), it simply accentuates the relationship between geography and gene flow. Slightly different implications emerge from imprinting on a kind of habitat, host, or resource because then the environmental influence on gene flow is independent of geography. Some phytophagous insects imprint on their 
host plant species based on chemical cues [102], and nestparasitic indigobirds imprint on their hosts [103]. In these examples, phenotypic plasticity helps maintain similarity between maternal and offspring environments, which is important both for maintaining phenotypic similarity and consistency of selection on parents and offspring. However, it is not always obvious that habitat or resource imprinting should directly affect mating preferences. In the Vidua indigobirds, there is an imprinting effect of host song, such that nest parasites raised by the same host species tend to mate assortatively owing to learned elements of their own songs and preferences $[103,104]$. In many phytophagous insects, mating occurs on or near the host plant $[102,105]$, making plant choice a "magic trait" simultaneously effecting ecological and sexual differentiation [31]. As with genetically determined traits, phenotypic plasticity of traits directly linked to both ecological adaptation and assortative mating is most likely to contribute to ecological speciation.

Environmental effects on traits directly involved in sexual selection are not unusual. Expression of pigments, pheromones, and other displays can depend on diet, condition, or experience $[19,106]$. For example, premating isolation is induced by larval host plant differences in Drosophila mojavensis, because the chemical properties of their cuticular hydrocarbons (important contact pheromones) are strongly influenced by diet [107, 108]. Sharon et al. [109] recently showed that mate choice in $D$. melanogaster can be modified by symbiotic gut bacteria. Flies raised on a high-starch medium had microbiota dominated by Lactobacillus plantarum, which was only a minor constituent of the microbiota of flies raised on a standard cornmeal-molasses medium. Sharon et al. [109] found that the differences in bacterial composition can affect cuticular hydrocarbon levels, providing a probable mechanism affecting mate choice.

In Sockeye Salmon (Oncorhynchus nerka), postmating isolation might be caused by sexual selection on diet-derived coloration. Anadromous sockeye sequester carotenoids from crustaceans consumed in the ocean and use them to express their brilliant red mating colors. The nonanadromous morph (kokanee) expresses equally bright red mating color on a diet with much less carotenoids, an example of countergradient variation [110]. Anadromous morphs and hybrids raised in the freshwater habitat of the kokanee (lowcarotenoid diet) underexpress the red pigment and probably suffer reduced mating success as a consequence $[111,112]$.

The actual effect of phenotypic plasticity on gene flow depends on environmental similarity between parents and offspring. Environmentally induced mate discrimination will have little or no hindrance on gene flow unless it also affects the phenotypes and/or environments of the offspring. For example, imagine a phytophagous insect with environmentally induced contact pheromones causing perfect assortative mating between individuals raised on the same host plant. If there are no differences in host choice, then the offspring of each mating type are equally likely to grow up on each plant and therefore have no tendency to develop the same pheromone profile as their parents (Figure 5). In this hypothetical case, there is free gene flow despite assortative mating of phenotypes.
Environmentally induced differences in habitat choice reduce gene flow when individuals are more likely to mate with other individuals using the same habitat, and offspring are more likely to grow up in habitats similar to those of their parents. If habitat choice is entirely determined by the individual's environment (i.e., if there is no tendency for the offspring of immigrants to return to their parent's original habitat), then the effect is genetically identical to a geographic barrier. Either way we can describe the system in terms of populations of individuals or gametes with some probability $(m)$ of "moving" from their natal population to breed in a different population. In the simple case of two environments and nonoverlapping generations, the expected frequency of an allele in generation $t$ in environment $i$ is a weighted average of the frequencies in environments $i$ and $j$ in generation $t-1$ [113]:

$$
p_{i(t)}=(1-m) p_{i(t-1)}+m p_{j(t-1)} .
$$

It makes no difference whether $m$ is determined by geography or environmental induction as long as there are not heritable differences in $m$ among individuals within a habitat. More generally, geographic or spatial covariance is a special case of environmental similarity, and the extensive knowledge from decades of conceptual and mathematical modeling of gene flow's effects on adaptation and speciation [30, $31,87,114,115]$ can be extended directly to include this kind of phenotypic plasticity. In particular, we might expect environmentally induced restrictions on gene flow to facilitate the evolution of postzygotic incompatibilities (both environment-dependent and -independent selection against hybrids) and genetic assimilation of behavioral reproductive barriers (habitat and mate choice), but also to lessen the potential for selective reinforcement of assortative mating (just as adaptive plasticity lessens divergent selection on ecological phenotypes).

\section{Conclusions and Future Directions}

Opinions still seem to outnumber data about the impact of environmental induction and plasticity on evolution, but substantial progress has been made in the last 20 years $[23,64]$. Plasticity appears to be a common if not universal feature of developmental systems and should not be ignored. Plasticity and environmental effects were once black boxes, ignored by some, uncritically promoted as threats to evolutionary theory by others. But theoretical and empirical investigations have increasingly shed light on how plasticity evolves and interacts with natural selection. Whether developmental plasticity rivals mutation as a source of quantitative or qualitative change (the evolution of "novelty") remains contentious $[17,80,116]$. However, to the extent that speciation is defined by genetic divergence, genes will not be displaced from their central role in the study of speciation. Phenotypic plasticity can promote or constrain adaptive evolution and ecological speciation. The effects of plasticity in a particular case, and whether there is an overall trend, are empirical questions.

Important theoretical challenges for understanding the importance of plasticity for adaptation and speciation 

A. 1st generation: Larvae on different host plants develop environmentally induced mating cues represented by red and blue.

B. Random dispersal results in equal frequencies on each host plant. Association between genotype and mating phenotype persists.

C. 2nd generation: Larvae produced by assortative mating deviate from HardyWeinberg, but develop environmentally induced mating cues.

D. Random dispersal maintains equal frequencies. There is no association between genotype and mating phenotype

E. 3rd generation: Hardy-Weinberg genotype proportions are expected for larvae on each host despite assortative mating by environmentally induced phenotype.
Host 1
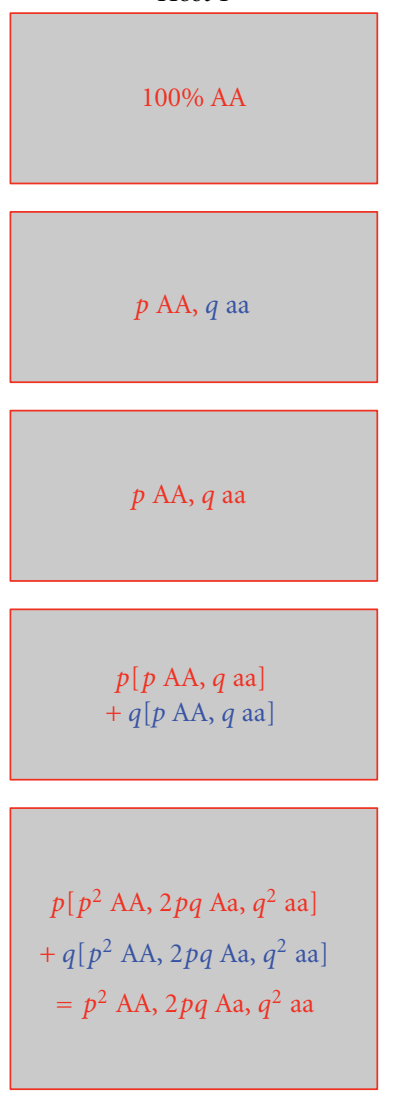

Host 2

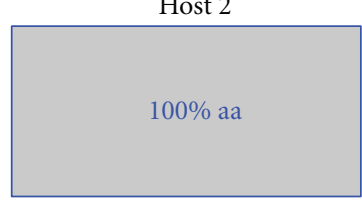

$p$ AA, $q$ aa
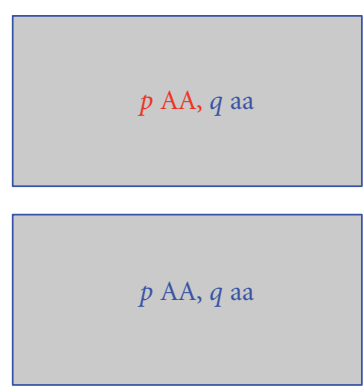

$p[p \mathrm{AA}, q$ aa $]$

$+q[p \mathrm{AA}, q \mathrm{aa}]$

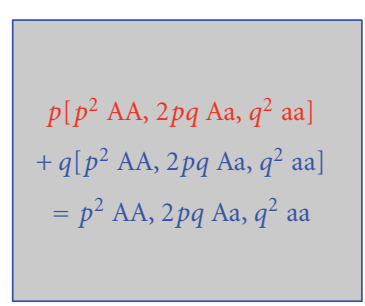

FIGURE 5: Free gene flow despite assortative mating. If mating cues are environmentally induced but there is no habitat choice, host-associated populations will not be genetically differentiated, and it takes two generations to establish Hardy-Weinberg equilibrium. If the host densities are $p$ and $1-p=q$, a locus that is fixed for different alleles on different hosts has allele frequencies $p$ and $q$. At first, genotypes are perfectly associated with the host-induced mating cue, but random dispersal eliminates that association in one generation if there are no other factors maintaining covariance between parent and offspring phenotype (e.g., maternal effects or divergent selection). Once genotype frequencies are equalized between phenotypes, mating within phenotypes establishes Hardy-Weinberg genotype proportions [61]. Although imagining a locus with complete differentiation makes for the simplest illustration, the result is completely general for any allele frequency. If we somehow know what alleles have ancestors in each habitat in generation 0 , the result is that it takes just two generations to completely randomize that ancestry, regardless of whether or not the alleles are actually different by state.

include the extension of models (such as [36]) to incorporate nonadaptive plasticity and countergradient variation, further investigation of how costs of plasticity affect genetic assimilation and reproductive isolation, and careful examination of what kinds of environmental effects on phenotype can be considered equivalent to geographic restrictions of gene flow (environmental effects on dispersal) [59, 93, 94, 114]. Not all environmental effects on mate choice will affect gene flow (Figure 5). However, maternal effects and factors that promote environmental similarity between relatives seem particularly likely to reduce gene flow and promote adaptive divergence. Incorporation of such effects into the classic models of local adaptation [87] and ecological speciation [31] might bring substantial clarification to the subject. Moreover, how environmentally induced barriers to gene flow might affect the evolution of constitutive genetic barriers has not been explored. Should we expect environmental effects on assortative mating to slow the evolution of genetic barriers to gene flow? Finally, virtually no attention has been given to the relationship between plasticity and postzygotic isolation. Key questions include how might plasticity affect ecologically based selection on hybrids, and are highly plastic developmental pathways good or bad candidates for involvement in postzygotic developmental incompatibilities?

Addressing empirical challenges might best begin by establishing criteria for recognizing plasticity as a causal factor in speciation or adaptive radiation. West-Eberhard [15] and Pfennig and McGee [117] suggested that associations between intraspecific plasticity and species diversity support a role for plasticity in promoting adaptive radiation. Examples like the tiger salamander radiation (Figure 1) and others [117], where an intraspecific polyphenism parallels a repeated pattern of interspecific divergence, are consistent with adaptive plasticity as an origin of ecological divergence between species. More confirmatory evidence for a causal role of plasticity in adaptive divergence (the first component of ecological speciation) would come from testing the strengths of tradeoffs, costs of plasticity, and selection against 
immigrants and hybrids. Effects of plasticity on reproductive isolation (the second component of ecological speciation) are illustrated in studies like those of parasitic indigobirds $[103,104]$ and Drosophila [107-109] on environmentally induced barriers to gene flow. We presently know little of the prevalence of this phenomenon or whether it is often strong enough to facilitate substantial genetic differentiation. Field studies are needed to document the prevalence and strength, in nature, of costs of plasticity, evolvability of reaction norms, and environmental effects on reproductive isolation. The principle illustrated in Figure 5 should be considered in the design and interpretation of experiments. In order for plasticity of mating behaviour to directly impact gene flow, there must be some factor maintaining similarity of environmental effects across generations. Finally, developmental genetic studies are needed to assess whether genes causing postzygotic or prezygotic isolation are often involved in highly plastic developmental pathways.

The theoretical and empirical foundations of speciation research are very strong in terms of genetics and geography [29-31, 115]. The roles of ecology, environment, and development are prominent among the remaining frontiers $[27,36,52]$. Further research integrating genetics, ecology, and development promises great gains in understanding the origins of biological diversity.

\section{Acknowledgments}

The author thanks A. Hendry and two reviewers for their very helpful comments on an earlier draft of this paper. His knowledge and ideas on the subject, and their presentation herein, were greatly improved by discussions with J. Fordyce and X. Thibert-Plante. Research relevant to this paper has been supported by the United States National Science Foundation (DEB-0516475 and DEB-1011216).

\section{References}

[1] S. Wright, "Evolution in Mendelian populations," Genetics, vol. 16, no. 2, pp. 97-159, 1931.

[2] S. C. Stearns, "The role of development in the evolution of life histories," in Evolution and Development, J. T. Bonner, Ed., pp. 237-258, Springer, Berlin, Germany, 1982.

[3] D. A. Levin, "Plasticity, canalization and evolutionary stasis in plants," in Plant Population Ecology, A. J. Davy, M. J. Hutchings, and A. R. Watkinson, Eds., pp. 35-45, Blackwell Scientific, 1988.

[4] J. Clausen, "Principles for a joint attack on evolutionary problems," in Proceedings of the 6th International Congress of Genetics, D. F. Jones, Ed., vol. 2, pp. 21-23, Brooklyn Botanic Garden, Ithaca, NY, USA, 1932.

[5] R. K. Clements, J. M. Baskin, and C. C. Baskin, "The comparative biology of the two closely-related species Penstemon tenuiflorus Pennell and P. hirsutus (L.) Willd. (Scrophulariaceae, section Graciles): I. Taxonomy and geographical distribution," Castanea, vol. 63, no. 2, pp. 138-153, 1998.

[6] P. B. Reich, I. J. Wright, J. Cavender-Bares et al., "The evolution of plant functional variation: traits, spectra, and strategies," International Journal of Plant Sciences, vol. 164, no. 3, pp. S143-S164, 2003.
[7] U. Dieckmann and M. Heino, "Probabilistic maturation reaction norms: their history, strengths, and limitations," Marine Ecology-Progress Series, vol. 335, pp. 253-269, 2007.

[8] M. G. Palacios, A. M. Sparkman, and A. M. Bronikowski, "Developmental plasticity of immune defence in two lifehistory ecotypes of the garter snake, thamnophis elegans - a common-environment experiment," Journal of Animal Ecology, vol. 80, no. 2, pp. 431-437, 2011.

[9] J. M. Baldwin, "A new factor in evolution," American Naturalist, vol. 30, pp. 441-451, 1896.

[10] C. H. Waddington, "Canalization of development and the inheritance of acquired characters," Nature, vol. 150, no. 3811, pp. 563-565, 1942.

[11] C. H. Waddington, "Genetic assimilation of acquired characters," Evolution, vol. 7, no. 4676, pp. 118-126, 1953.

[12] Schmalhausen II, Factors of Evolution, Blakiston, Philadelphia, Pa, USA, 1949.

[13] M. J. West-Eberhard, "Alternative adaptations, speciation, and phylogeny," Proceedings of the National Academy of Sciences of the United States of America, vol. 83, no. 5, pp. 1388-1392, 1986.

[14] M. J. West-Eberhard, "Phenotypic plasticity and the origins of diversity," Annual review of Ecology and Systematics, vol. 20, pp. 249-278, 1989.

[15] M. J. West-Eberhard, Developmental Plasticity and Evolution, Oxford University Press, Oxford, UK, 2003.

[16] C. D. Schlichting, "The role of phenotypic plasticity in diversification," in Phenotypic Plasticity: Functional and Conceptual Approaches, T. J. DeWitt and S. M. Scheiner, Eds., pp. 191-200, Oxford University Press, 2004.

[17] M. J. West-Eberhard, "Developmental plasticity and the origin of species differences," Proceedings of the National Academy of Sciences of the United States of America, vol. 102, no. 1, pp. 6543-6549, 2005.

[18] G. F. Grether, "Environmental change, phenotypic plasticity, and genetic compensation," The American Naturalist, vol. 166, no. 4, pp. E115-123, 2005.

[19] T. D. Price, "Phenotypic plasticity, sexual selection and the evolution of colour patterns," Journal of Experimental Biology, vol. 209, no. 12, pp. 2368-2376, 2006.

[20] E. Crispo, "The Baldwin effect and genetic assimilation: revisiting two mechanisms of evolutionary change mediated by phenotypic plasticity," Evolution, vol. 61, no. 11, pp. 24692479, 2007.

[21] E. Crispo, "Modifying effects of phenotypic plasticity on interactions among natural selection, adaptation and gene flow," Journal of Evolutionary Biology, vol. 21, no. 6, pp. 1460 1469, 2008.

[22] C. K. Ghalambor, J. K. McKay, S. P. Carroll, and D. N. Reznick, "Adaptive versus non-adaptive phenotypic plasticity and the potential for contemporary adaptation in new environments," Functional Ecology, vol. 21, no. 3, pp. 394407, 2007.

[23] S. F. Gilbert and D. Epel, Ecological Developmental Biology: Integrating Epigenetics, Medicine, and Evolution, Sinauer Associates, 2009.

[24] G. Fusco and A. Minelli, "Phenotypic plasticity in development and evolution: facts and concepts," Philosophical Transactions of the Royal Society B, vol. 365, no. 1540, pp. 547-556, 2010.

[25] T. Schwander and O. Leimar, "Genes as leaders and followers in evolution," Trends in Ecology and Evolution, vol. 26, no. 3, pp. 143-151, 2011. 
[26] T. Uller and H. Helantera, "When are genes 'leaders' or 'followers' in evolution?" Trends in Ecology and Evolution, vol. 26, no. 9, pp. 435-436, 2011.

[27] D. W. Pfennig, M. A. Wund, E. C. Snell-Rood, T. Cruickshank, C. D. Schlichting, and A. P. Moczek, "Phenotypic plasticity's impacts on diversification and speciation," Trends in Ecology and Evolution, vol. 25, no. 8, pp. 459-467, 2010.

[28] H. D. Rundle and P. Nosil, "Ecological speciation," Ecology Letters, vol. 8, no. 3, pp. 336-352, 2005.

[29] M. Turelli, N. H. Barton, and J. A. Coyne, "Theory and speciation," Trends in Ecology and Evolution, vol. 16, no. 7, pp. 330-343, 2001.

[30] J. A. Coyne and H. A. Orr, Speciation, Sinauer Associates, Sunderland, Mass, USA, 2004.

[31] S. Gavrilets, Fitness Landscapes and the Origin of Species, Princeton University Press, Princeton, NJ, USA, 2004.

[32] T. D. Price, A. Qvarnstrom, and D. E. Irwin, "The role of phenotypic plasticity in driving genetic evolution," Proceedings of the Royal Society B, vol. 270, no. 1523, pp. 1433-1440, 2003.

[33] R. Lande, "Adaptation to an extraordinary environment by evolution of phenotypic plasticity and genetic assimilation," Journal of Evolutionary Biology, vol. 22, no. 7, pp. 1435-1446, 2009.

[34] D. A. Levin, "Flowering-time plasticity facilitates niche shifts in adjacent populations," New Phytologist, vol. 183, no. 3, pp. 661-666, 2009.

[35] R. Svanbäck, M. Pineda-Krch, and M. Doebeli, "Fluctuating population dynamics promotes the evolution of phenotypic plasticity," American Naturalist, vol. 174, no. 2, pp. 176-189, 2009.

[36] X. Thibert-Plante and A. P. Hendry, "The consequences of phenotypic plasticity for ecological speciation," Journal of Evolutionary Biology, vol. 24, no. 2, pp. 326-342, 2011.

[37] M. D. Herron and M. Doebeli, "Adaptive diversification of a plastic trait in a predictably fluctuating environment," Journal of Theoretical Biology, vol. 285, pp. 58-68, 2011.

[38] D. J. Funk, "Of 'host forms' and host races: terminological issues in ecological speciation," International Journal of Ecology, vol. 2012, Article ID 506957, 8 pages, 2012.

[39] J. K. Conner and D. L. Hartl, A Primer of Ecological Genetics, Sinauer Associates, Sunderland, Mass, USA, 2004.

[40] D. J. Futuyma, Evolution, Sinauer Associates, Sunderland, Mass, USA, 2nd edition, 2009.

[41] D. O. Conover and E. T. Schultz, "Phenotypic similarity and the evolutionary significance of countergradient variation," Trends in Ecology and Evolution, vol. 10, no. 6, pp. 248-252, 1995.

[42] J. A. Fordyce, "The evolutionary consequences of ecological interactions mediated through phenotypic plasticity," Journal of Experimental Biology, vol. 209, no. 12, pp. 2377-2383, 2006.

[43] G. R. Price, "Selection and covariance," Nature, vol. 227, no. 5257, pp. 520-521, 1970.

[44] J. A. Endler, Natural Selection in the Wild, Princeton University Press, Princeton, NJ, USA, 1986.

[45] S. Via, "The evolution phenotypic plasticity: what do we really know?" in Ecological Genetics, L. A. Real, Ed., pp. 3557, Princeton University Press, 1994.

[46] G. L. Bush, "Reply [to M. Claridge] from G. L. Bush," Trends in Ecology \& Evolution, vol. 10, no. 1, p. 38, 1995.

[47] M. Claridge, "Species and speciation," Trends in Ecology \& Evolution, vol. 10, no. 1, p. 38, 1995.
[48] G. G. Simpson, Tempo and Mode in Evolution, Columbia University Press, New York, NY, USA, 1944.

[49] S. J. Gould, The Structure of Evolutionary Theory, Harvard University Press, Cambridge, Mass, USA, 2002.

[50] T. F. Stuessy, G. Jakubowsky, R. S. Gomez et al., "Anagenetic evolution in island plants," Journal of Biogeography, vol. 33, no. 7, pp. 1259-1265, 2006.

[51] A. P. Hendry, "Ecological speciation! or the lack thereof?" Canadian Journal of Fisheries and Aquatic Sciences, vol. 66, no. 8, pp. 1383-1398, 2009.

[52] D. Schluter, "Evidence for ecological speciation and its alternative," Science, vol. 323, no. 5915, pp. 737-741, 2009.

[53] E. Mayr, Systematics and the Origin of Species from the Viewpoint of a Zoologist, Columbia University Press, New York, NY, USA, 1942.

[54] R. Matsuda, "The evolutionary process in talitrid amphipods and salamanders in changing environments, with a discussion of "genetic assimilation" and some other evolutionary concepts," Canadian Journal of Zoology, vol. 60, no. 5, pp. 733-749, 1982.

[55] H. B. Shaffer and S. R. Voss, "Phylogenetic and mechanistic analysis of a developmentally integrated character complex: alternate life history modes in ambystomatid salamanders," American Zoologist, vol. 36, no. 1, pp. 24-35, 1996.

[56] S. R. Voss and H. B. Shaffer, "Adaptive evolution via a major gene effect: paedomorphosis in the Mexican axolotl," Proceedings of the National Academy of Sciences of the United States of America, vol. 94, no. 25, pp. 14185-14189, 1997.

[57] H. B. Shaffer, "Evolution in a paedomorphic lineage. I. An electrophoretic analysis of the Mexican ambystomatid salamanders," Evolution, vol. 38, pp. 1194-1206, 1984.

[58] H. B. Shaffer and M. L. Mcknight, "The polytypic species revisited: genetic differentiation and molecular phylogenetics of the tiger salamander Ambystoma tigrinum (Amphibia: Caudata) complex," Evolution, vol. 50, no. 1, pp. 417-433, 1996.

[59] S. Gavrilets and A. Vose, "Case studies and mathematical models of ecological speciation. 2. Palms on an oceanic island," Molecular Ecology, vol. 16, no. 14, pp. 2910-2921, 2007.

[60] V. Savolainen, M. C. Anstett, C. Lexer et al., "Sympatric speciation in palms on an oceanic island," Nature, vol. 441, no. 7090, pp. 210-213, 2006.

[61] G. H. Hardy, "Mendelian proportions in a mixed population," Science, vol. 28, no. 706, pp. 49-50, 1908.

[62] D. Schluter, "Ecology and the origin of species," Trends in Ecology and Evolution, vol. 16, no. 7, pp. 372-380, 2001.

[63] K. Räsänen and A. P. Hendry, "Disentangling interactions between adaptive divergence and gene flow when ecology drives diversification," Ecology Letters, vol. 11, no. 6, pp. 624636, 2008.

[64] T. J. DeWitt and S. M. Scheiner, "Phenotypic variation from single genotypes: a primer," in Phenotypic Plasticity: Functional and Conceptual Approaches, T. J. DeWitt and S. M. Scheiner, Eds., pp. 1-9, Oxford University Press, New York, NY, USA, 2004.

[65] S. Via and R. Lande, "Genotype-environment interaction and the evolution of phenotypic plasticity," Evolution, vol. 39, no. 3, pp. 505-522, 1985.

[66] L. Zhivotovsky, M. Feldman, and A. Bergman, "On the evolution of phenotypic plasticity in a spatially heterogeneous environment," Evolution, vol. 50, no. 2, pp. 547-558, 1996.

[67] S. Sultan and H. G. Spencer, "Metapopulation structure favors plasticity over local adaptation," American Naturalist, vol. 160, no. 2, pp. 271-283, 2002. 
[68] J. Hollander, "Testing the grain-size model for the evolution of phenotypic plasticity," Evolution, vol. 62, no. 6, pp. 1381$1389,2008$.

[69] J. B. S. Haldane, "A mathematical theory of natural and artificial selection-part VI. Isolation," Proceedings of the Cambridge Philosophical Society, vol. 26, pp. 220-230, 1930.

[70] M. G. Bulmer, "Multiple niche polymorphism," American Naturalist, vol. 106, pp. 254-257, 1972.

[71] M. Slatkin, "Gene flow and the geographic structure of natural populations," Science, vol. 236, no. 4803, pp. 787-792, 1987.

[72] W. R. Rice and E. E. Hostert, "Laboratory experiments on speciation: what have we learned in 40 years?" Evolution, vol. 47, no. 6, pp. 1637-1653, 1993.

[73] S. Gavrilets, "Models of speciation: what have we learned in 40 years?" Evolution, vol. 57, no. 10, pp. 2197-2215, 2003.

[74] R. D. Holt, "On the evolutionary ecology of species' ranges," Evolutionary Ecology Research, vol. 5, no. 2, pp. 159-178, 2003.

[75] D. Garant, S. E. Forde, and A. P. Hendry, "The multifarious effects of dispersal and gene flow on contemporary adaptation," Functional Ecology, vol. 21, no. 3, pp. 434-443, 2007.

[76] T. J. Kawecki, "Adaptation to marginal habitats," Annual Review of Ecology, Evolution, and Systematics, vol. 39, pp. 321342, 2008.

[77] S. Via, "Genetic constraints on the evolution of phenotypic plasticity," in Genetic Constraints on Adaptive Evolution, V. Loeschcke, Ed., pp. 47-71, Springer, Boston, Mass, USA, 1987.

[78] S. Via, R. Gomulkiewicz, G. de Jong, S. M. Scheiner, C. D. Schlichting, and P. H. Van Tienderen, "Adaptive phenotypic plasticity: consensus and controversy," Trends in Ecology and Evolution, vol. 10, no. 5, pp. 212-217, 1995.

[79] A. Romero and S. M. Green, "The end of regressive evolution: examining and interpreting the evidence from cave fishes," Journal of Fish Biology, vol. 67, no. 1, pp. 3-32, 2005.

[80] M. Pigliucci, C. J. Murren, and C. D. Schlichting, "Phenotypic plasticity and evolution by genetic assimilation," Journal of Experimental Biology, vol. 209, no. 12, pp. 23622367, 2006.

[81] R. B. Langerhans and T. J. DeWitt, "Plasticity constrained: over-generalized induction cues cause maladaptive phenotypes," Evolutionary Ecology Research, vol. 4, no. 6, pp. 857870, 2002.

[82] F. C. James, "Environmental component of morphological differentiation in birds," Science, vol. 221, no. 4606, pp. 184 186, 1983.

[83] T. Steinger, B. A. Roy, and M. L. Stanton, "Evolution in stressful environments II: adaptive value and costs of plasticity in response to low light in Sinapis arvensis," Journal of Evolutionary Biology, vol. 16, no. 2, pp. 313-323, 2003.

[84] P. H. Van Tienderen, "Evolution of generalists and specialists in spatially heterogeneous environments," Evolution, vol. 45, no. 6, pp. 1317-1331, 1991.

[85] T. J. DeWitt, A. Sih, and D. S. Wilson, "Costs and limits of phenotypic plasticity," Trends in Ecology and Evolution, vol. 13, no. 2, pp. 77-81, 1998.

[86] T. B. Smith and S. Skulason, "Evolutionary significance of resource polymorphisms in fishes, amphibians, and birds," Annual Review of Ecology and Systematics, vol. 27, pp. 111133, 1996.

[87] T. Lenormand, "Gene flow and the limits to natural selection," Trends in Ecology and Evolution, vol. 17, no. 4, pp. 183$189,2002$.
[88] C. D. Schlichting and M. Pigliucci, Phenotypic Evolution: A Reaction Norm Perspective, Sinauer Associates, 1998.

[89] T. H. Bullock, "Compensation for temperature in the metabolism and activity of poikilotherms," Biological Reviews, vol. 30, pp. 311-342, 1955.

[90] R. Levins, "Thermal acclimation and heat resistance in Drosophila species," American Naturalist, vol. 103, pp. 483499, 1969.

[91] K. A. Berven, D. E. Gill, and S. J. Smithgill, "Countergradient selection in the green frog, rana clamitans," Evolution, vol. 33, pp. 609-623, 1979.

[92] M. Kirkpatrick and N. H. Barton, "Evolution of a species' range," American Naturalist, vol. 150, no. 1, pp. 1-23, 1997.

[93] B. M. Fitzpatrick, J. A. Fordyce, and S. Gavrilets, "What, if anything, is sympatric speciation?" Journal of Evolutionary Biology, vol. 21, no. 6, pp. 1452-1459, 2008.

[94] B. M. Fitzpatrick, J. A. Fordyce, and S. Gavrilets, "Pattern, process and geographic modes of speciation," Journal of Evolutionary Biology, vol. 22, no. 11, pp. 2342-2347, 2009.

[95] J. Antonovics, "Evolution in closely adjacent plant populations. X. Long-term persistence of prereproductive isolation at a mine boundary," Heredity, vol. 97, no. 1, pp. 33-37, 2006.

[96] M. C. Hall and J. H. Willis, "Divergent selection on flowering time contributes to local adaptation in Mimulus guttatus populations," Evolution, vol. 60, no. 12, pp. 2466-2477, 2006.

[97] J. B. Beltman and P. Haccou, "Speciation through the learning of habitat features," Theoretical Population Biology, vol. 67, no. 3, pp. 189-202, 2005.

[98] J. Beltman and J. A. Metz, "Speciation: more likely through a genetic or through a learned habitat preference?" Proceedings of the Royal Society B, vol. 272, no. 1571, pp. 1455-1463, 2005.

[99] J. M. Davis and J. A. Stamps, "The effect of natal experience on habitat preferences," Trends in Ecology and Evolution, vol. 19, no. 8, pp. 411-416, 2004.

[100] A. P. Hendry, V. Castric, M. T. Kinnison, and T. P. Quinn, "The evolution of philopatry and dispersal: homing versus straying in salmonids," in Evolution illuminated: Salmon and their relatives, A. P. Hendry and S. C. Stearns, Eds., pp. 52-91, Oxford University Press, Oxford, UK, 2004.

[101] N. Vallin and A. Qvarnstrom, "Learning the hard way: imprinting can enhance enforced shifts in habitat choice," International Journal of Ecology, vol. 2011, Article ID 287532, 7 pages, 2011.

[102] E. A. Bernays and R. F. Chapman, Host-Plant Selection by Phytophagous Insects, Chapman and Hall, New York, NY, USA, 1994.

[103] M. D. Sorenson, K. M. Sefc, and R. B. Payne, "Speciation by host switch in brood parasitic indigobirds," Nature, vol. 424, no. 6951, pp. 928-931, 2003.

[104] C. N. Balakrishnan, K. M. Sefc, and M. D. Sorenson, "Incomplete reproductive isolation following host shift in brood parasitic indigobirds," Proceedings of the Royal Society B, vol. 276, no. 1655, pp. 219-228, 2009.

[105] S. H. Berlocher and J. L. Feder, "Sympatric speciation in phytophagous insects: moving beyond controversy?" Annual Review of Entomology, vol. 47, pp. 773-815, 2002.

[106] H. Knüttel and K. Fiedler, "Host-plant-derived variation in ultraviolet wing patterns influences mate selection by male butterflies," Journal of Experimental Biology, vol. 204, no. 14, pp. 2447-2459, 2001.

[107] M. D. Stennett and W. J. Etges, "Premating isolation is determined by larval rearing substrates in cactophilic Drosophila mojavensis. III. Epicuticular hydrocarbon variation is determined by use of different host plants in Drosophila mojavensis 
and Drosophila arizonae," Journal of Chemical Ecology, vol. 23, no. 12, pp. 2803-2824, 1997.

[108] W. J. Etges, C. L. Veenstra, and L. L. Jackson, "Premating isolation is determined by larval rearing substrates in cactophilic Drosophila mojavensis. VII. Effects of larval dietary fatty acids on adult epicuticular hydrocarbons," Journal of Chemical Ecology, vol. 32, pp. 2629-2646, 2006.

[109] G. Sharon, D. Segal, J. M. Ringo, A. Hefetz, I. ZilberRosenberg, and E. Rosenberg, "Commensal bacteria play a role in mating preference of Drosophila melanogaster," Proceedings of the National Academy of Sciences of the United States of America, vol. 107, no. 46, pp. 20051-20056, 2010.

[110] J. K. Craig and C. J. Foote, "Countergradient variation and secondary sexual color: phenotypic convergence promotes genetic divergence in carotenoid use between sympatric anadromous and nonanadromous morphs of sockeye salmon (Onchorhynchus nerka)," Evolution, vol. 55, pp. 380391, 2001.

[111] C. J. Foote, G. S. Brown, and C. W. Hawryshyn, "Female colour and male choice in sockeye salmon: implications for the phenotypic convergence of anadromous and nonanadromous morphs," Animal Behaviour, vol. 67, no. 1, pp. 69-83, 2004.

[112] J. K. Craig, C. J. Foote, and C. C. Wood, "Countergradient variation in carotenoid use between sympatric morphs of sockeye salmon (Oncorhynchus nerka) exposes nonanadromous hybrids in the wild by their mismatched spawning colour," Biological Journal of the Linnean Society, vol. 84, no. 2, pp. 287-305, 2005.

[113] D. L. Hartl and A. G. Clark, Principles of Population Genetics, Sinauer Associates, Sunderland, Mass, USA, 3rd edition, 1997.

[114] M. Kirkpatrick and V. Ravigné, "Speciation by natural and sexual selection: models and experiments," American Naturalist, vol. 159, pp. S22-S35, 2002.

[115] D. I. Bolnick and B. M. Fitzpatrick, "Sympatric speciation: models and empirical evidence," Annual Review of Ecology, Evolution, and Systematics, vol. 38, pp. 459-487, 2007.

[116] G. de Jong, "Evolution of phenotypic plasticity: patterns of plasticity and the emergence of ecotypes," New Phytologist, vol. 166, no. 1, pp. 101-117, 2005.

[117] D. W. Pfennig and M. McGee, "Resource polyphenism increases species richness: a test of the hypothesis," Philosophical Transactions of the Royal Society B, vol. 365, no. 1540, pp. 577-591, 2010. 

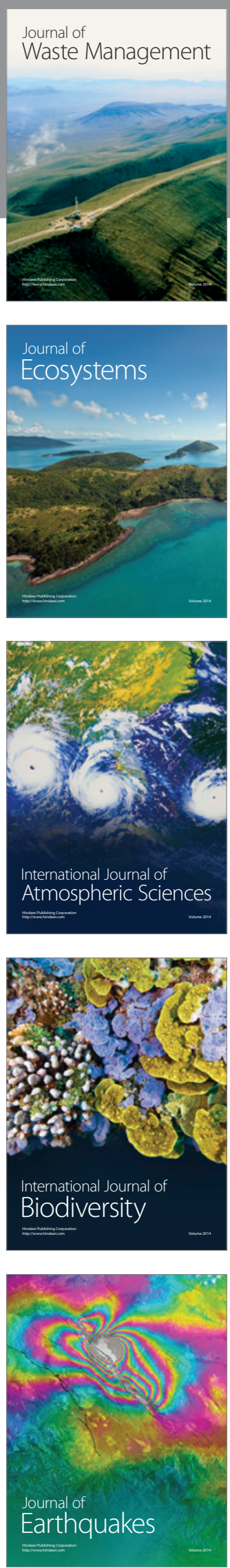
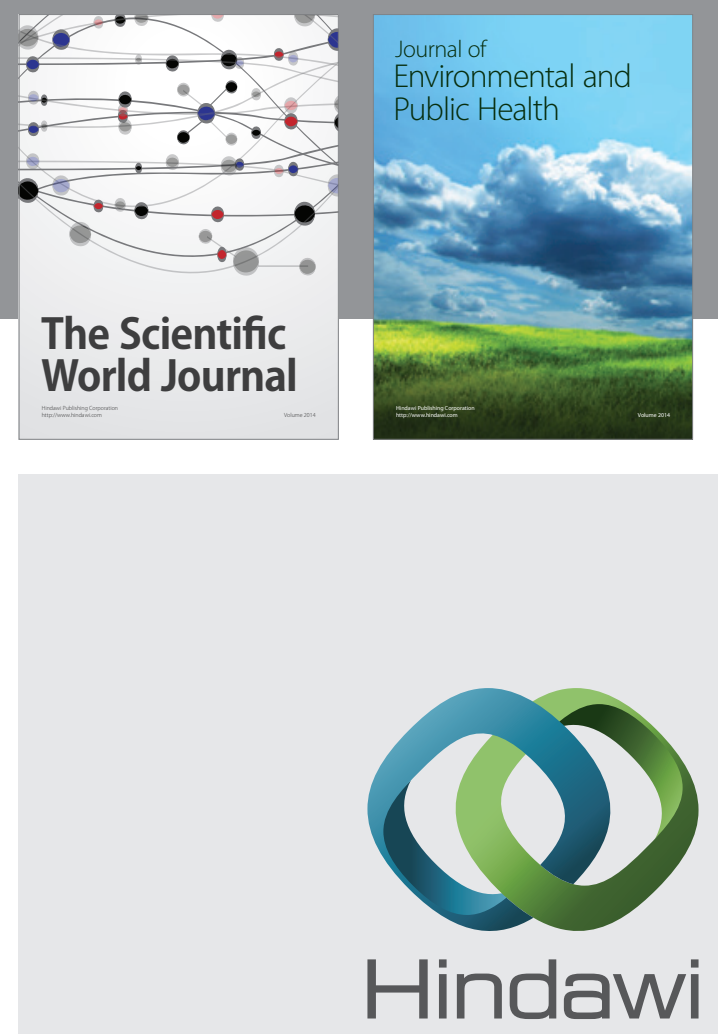

Submit your manuscripts at

http://www.hindawi.com
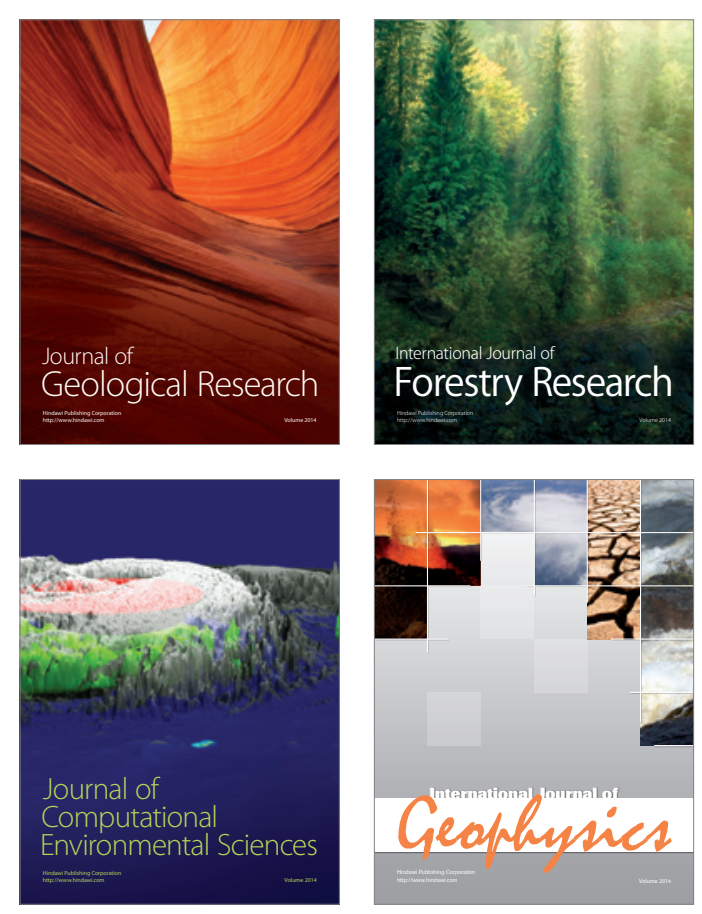
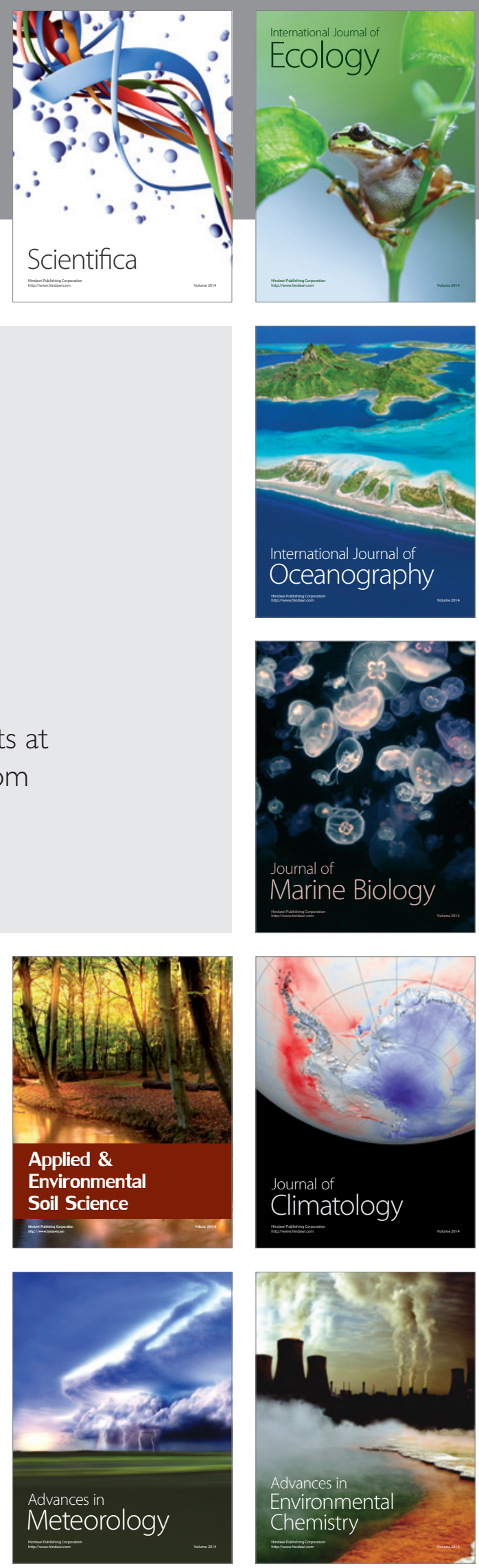